\title{
Porcine circovirus type 2 (PCV2) induces cell proliferation, fusion, and chemokine expression in swine monocytic cells in vitro
}

\author{
Yi-Chieh Tsai $^{1}$, Chian-Ren $\mathrm{JENG}^{1,2}$, Shih-Hsuan Hsiao ${ }^{1}$, Hui-Wen $\mathrm{CHANG}^{1}$, \\ Jiuan Judy LIU ${ }^{1}$, Chih-Cheng Chang ${ }^{3}$, Chun-Ming LIN $^{1}$, Mi-Yuan CHIA ${ }^{1}$, \\ Victor Fei $\mathrm{PANG}^{1,2 *}$ \\ ${ }^{1}$ Graduate Institute of Veterinary Medicine, School of Veterinary Medicine, National Taiwan University, No. 1, \\ Sec. 4, Roosevelt Rd., Taipei 106, Taiwan, Republic of China \\ ${ }^{2}$ Veterinary Hospital, College of Bio-Resources and Agriculture, National Taiwan University, No. 153, Sec. 3, \\ Kee-Loong Rd., Taipei 106, Taiwan, Republic of China \\ ${ }^{3}$ Department of Veterinary Medicine, National Chia Yi University, No. 300, University Rd., Chia Yi 600, Taiwan, \\ Republic of China
}

(Received 21 July 2009; accepted 21 May 2010)

\begin{abstract}
Granulomatous lymphadenitis is one of the pathognomonic lesions in post-weaning multisystemic wasting syndrome (PMWS)-affected pigs. This unique lesion has not been reported in direct association with viral infection in pigs. The objective of the present study was to evaluate whether porcine circovirus type 2 (PCV2) alone is able to induce functional modulation in porcine monocytic cells in vitro to elucidate its possible role in the development of granulomatous inflammation. It was found that the proliferation activity of blood monocytes (Mo) and monocyte-derived macrophages (MDM) was significantly enhanced by PCV2. During monocyte-macrophage differentiation, the PCV2 antigencontaining rate and formation of multinucleated giant cells (MGC) were significantly increased in MDM when compared to those in Mo. The MDM-derived MGC displayed a significantly higher PCV2 antigencontaining rate than did the mono-nucleated MDM. Supernatants from PCV2-inoculated MDM at $24 \mathrm{~h}$ post-inoculation induced an increased tendency of chemotactic activity for blood Mo. At the same inoculation time period, levels of mRNA expression of the monocytic chemokines, monocyte chemoattractant protein-1 and macrophage inflammatory protein-1, also significantly increased in PCV2-inoculated MDM. The results suggest that PCV2 alone may induce cell proliferation, fusion, and chemokine expression in swine monocytic cells. Thus, PCV2 itself may play a significant role in the induction of granulomatous inflammation in PMWS-affected pigs.
\end{abstract}

porcine circovirus type 2 / proliferation / multinucleated giant cell / chemotaxis / monocyte-derived macrophage

\section{INTRODUCTION}

Porcine circovirus type 2 (PCV2) is now recognised as the major causative agent of

\footnotetext{
* Corresponding author: pang@ntu.edu.tw
}

post-weaning multisystemic wasting syndrome (PMWS) [1, 35]. This syndrome characterised clinically by progressive weight loss and enlarged lymph nodes affects mainly 4- to $12-$ week-old weaned pigs although it also occurs in finishing pigs $[1,10]$. Microscopic lesions 
attributable to PMWS are primarily found in the lymphoid organs, liver, kidney, and lungs. The lymphoid tissue displays variable lymphoid depletion along with multifocal to diffuse infiltration of histiocytes and formation of multinucleated giant cells (MGC) that sometimes contain basophilic, grape-like, intracytoplasmic inclusions [35]. Whether PCV2 replicates in monocytic cells is still controversial, the monocyte/macrophage lineage cells are considered as the main target cells for PCV2 because PCV2 antigens and nucleic acid could be easily detected in these cells $[9,12,35]$. A unique histopathological change in the lymphoid tissues of PMWS-affected pigs is the granulomatous inflammation [20, 35]. This type of inflammation usually develops as a result of activated cell-mediated hypersensitivity due to the persistence of a non-degradable product [19]. The development of such a lesion requires recruitment, migration, and fusion of monocytes (Mo) and tissue macrophages. The serial events are regulated by complex interplays among invading organisms or prolonged antigenemia, macrophage activity, Th1 cell response, B cell over reactivity, and a vast array of biological mediators. Within the granulomatous lesion of lymph nodes from pigs naturally or experimentally infected by PCV2, it has been demonstrated that some of the cells could express monocyte chemoattractant protein-1 (MCP-1) $[21,22]$ and macrophage inflammatory protein-1 (MIP-1) [22]. These two cytokines are known to be important chemokines for the recruitment of Mo from the blood [28]. The progressive granulomatous inflammation has been speculated to be able to compromise organ functions and result in the PMWS-associated fatalities [23]. Thus, monocyte/macrophage infiltration has been suggested possibly related to the pathogenesis and progression of PMWS [21]. However, references regarding mechanisms of granulomatous inflammation formation provoked directly by PCV2 have seldom been discussed and reported. The objective of the present study was to evaluate the potential of PCV2 alone on the induction of proliferation, migration, and MGC formation of $\mathrm{Mo}$ and monocyte-derived macrophages (MDM) under in vitro conditions to elucidate the possible role of PCV2 on the development of granulomatous inflammation in vivo.

\section{MATERIALS AND METHODS}

\subsection{Experimental animals}

Twelve crossbred, healthy, conventional pigs, including 6 castrated males and 6 females, were used for serial blood collection from 10- to 14-weeks of age. All pigs had not received any vaccine when they were purchased. They were also tested negative for seral antibodies and viral nucleic acid for porcine respiratory and reproductive virus (PRRSV), PCV1, and PCV2 by indirect immunofluorescence assay (IFA) and RT-PCR, multiplex PCR [24] or nested PCR [25]. Aside from only 3 pigs, including 1 male and 2 females, that were used in the chemotaxis and monocytic chemokine expression experiments, 6 pigs with equal sex were used in all other experiments. Cells used in each experiment were collected from pigs at the same age. The animal use protocol was reviewed and approved by the Institutional Animal Care and Use Committee of National Taiwan University (Taiwan, Republic of China).

\subsection{Preparation of blood Mo and MDM}

Peripheral blood mononuclear cells (PBMC) were isolated from the heparinized (Leo Pharmaceutical, Ballerup, Denmark) jugular venous blood by density gradient centrifugation with Ficoll-Paque (Pharmacia, Uppsala, Sweden). The cells were resuspended in Roswell Park Memorial Institute (RPMI) medium (Gibco Laboratories, Grand Island, NY, USA) supplemented with $10 \%(\mathrm{v} / \mathrm{v})$ fetal bovine serum (FBS), $2 \mathrm{mM}$ L-glutamine (Sigma, St. Louis, MO, USA), $100 \mathrm{units} / \mathrm{mL}$ of penicillin (Sigma), and $100 \mu \mathrm{g} / \mathrm{mL}$ of streptomycin (Sigma) (RPMI-C). Non-adherent cells were removed following $2 \mathrm{~h}$ of incubation at $37{ }^{\circ} \mathrm{C}$ with $5 \% \mathrm{CO}_{2}$ in $75 \mathrm{~cm}^{2}$ flask (Corning-Costor, Acton, MA, USA). The adherent cells were either collected immediately by washing with ice cold RPMI containing $0.02 \%$ EDTA (Merck, Darmstadt, Germany) (RPMI-EDTA), designated as freshly collected Mo, or further incubated at $37^{\circ} \mathrm{C}$ in RPMI supplemented with $30 \%(\mathrm{v} / \mathrm{v})$ pooled heparinized porcine plasma free of PCV1, PCV2, and PRRSV. Following 
an additional $72 \mathrm{~h}$ of incubation, the cells collected were designated as MDM, as suggested by Basta et al. [2] based on phenotypic characteristics.

\subsection{Characterization of surface antigens expressed on Mo and MDM}

To characterize surface antigens expressed on different stages of blood-derived monocytic cells, Mo and MDM at $10^{6} / 100 \mu \mathrm{L}$ were stained with monoclonal antibodies (mAb) against SWC1 (K263.3D7, Serotec, Oxford, UK), a porcine monocyte marker [2]; SWC9 (PM 18-7, Serotec), a porcine macrophage marker [29]; MHCI (VMRD, Pullman, WA, USA); and MHCII (VMRD) and analyzed by flow cytometry. For single-antibody labelling, following staining with the above $\mathrm{mAb}$ for $20 \mathrm{~min}$ at $4{ }^{\circ} \mathrm{C}$, the Mo or MDM were then washed with flow buffer (PBS containing $0.1 \%$ sodium azide and 2\% FBS) and resuspended in $20 \mu \mathrm{L}$ of goat anti-mouse IgGsFITC (Serotec) for another $20 \mathrm{~min}$ at $4{ }^{\circ} \mathrm{C}$. The cells were then washed with flow buffer and analyzed by a FACSCalibur (BD Biosciences, Sunnyvale, CA, USA) with the CellQuest software (BD Biosciences). An area containing the major grouped cells was defined as R1 in the forward scatter/side scatter (FSC/SSC) plots. A total of 10000 events were recorded for each sample at the R1 area. For fluorescence analysis, the intensity of the green fluorescence (FITC-FL1 detection) was obtained from the R1 area. Under the same voltage, negative control cells were placed below $10^{1}$.

\subsection{Virus}

The PCV2 isolate and viral propagation were the same as those described previously $[6,7]$. The titer of PCV2 stock used in the present study was $10^{6}$ $\mathrm{TCID}_{50} / \mathrm{mL}$.

\subsection{Experimental design}

For each assay, Mo and/or MDM were inoculated with PCV2 at a multiplicity of infection (m.o.i.) of 1 or with an equal volume of medium in 24-well culture plates (Corning-Costar). At 24-120 h post-inoculation (HPI), Mo and/or MDM from each group was obtained and subjected to the analysis of total cell number and survival rate by trypan blue dye exclusion assay. The PCV2 antigen-containing rate, cell fusion rate, cell migration, and chemokine (MIP-1 and
MCP-1) mRNA expression were analyzed at 24 HPI by IFA light microscopy, Transwell ${ }^{\circledR}$ migration assay, and semi-quantitative RT-PCR, respectively.

\subsection{Proliferation ability and survival rate of PCV2- and mock-inoculated Mo and MDM}

Freshly isolated Mo and MDM were seeded onto 24-well culture plates (Corning-Corsta). At each designated time point after PCV2-inoculation, the culture supernatant from each well was removed and the adherent Mo and MDM were washed out from the culture plate by incubating with $1 \mathrm{~mL}$ of ice cold RPMI-EDTA for $10 \mathrm{~min}$. The cells were then collected by centrifugation at $800 \mathrm{rpm}, 4{ }^{\circ} \mathrm{C}$ for $10 \mathrm{~min}$ and re-suspended in $1 \mathrm{~mL}$ of RPMI-C. The proliferation ability was determined by counting the total number of viable and dead cells by trypan blue dye exclusion assay on a hemocytometer at each designated time point by light microscopy. The survival rate was calculated as the number of viable cells divided by the total number of viable and dead cells at each time point.

\subsection{Cell fusion rate of PCV2- and mock-inoculated Mo and MDM}

The MGC were defined as cells containing two or more nuclei. Using the same preparation as described above, the fusion rates of Mo and MDM were determined as reported by Most et al. [32] by counting the number of nuclei within MGC in a given area divided by the total number of nuclei in the same area, where fusion rate $(\%)=[($ number of nuclei within $\mathrm{MGC}) /$ (total number of nuclei counted) $\times 100 \%$. A total of 300-400 nuclei from 10 randomly selected fields were counted for in each preparation.

\subsection{PCV2 antigen-containing rate of PCV2- and mock-inoculated Mo and MDM}

Freshly isolated Mo and MDM were seeded onto 24-well culture plates (Corning-Costar) containing a sterile $12 \mathrm{~mm}$ coverslip in each well and incubated with PCV2 at an m.o.i. of 1 for $24 \mathrm{~h}$. The coverslips were then collected, washed with PBS, and fixed in $80 \%$ acetone at $-20{ }^{\circ} \mathrm{C}$ for $10 \mathrm{~min}$. After being washed with PBS and desiccated at room temperature, the coverslips were incubated with a 1:150 
diluted hyperimmune swine anti-PCV2 antiserum at $37^{\circ} \mathrm{C}$ for $1 \mathrm{~h}$ followed by a goat anti-porcine IgGFITC (Serotec) and counterstained with 1\% Hoechst 33258 (Sigma). In each preparation, cells with green fluorescence were counted and the percentages of positivity were calculated by counting 300 cells out of 10 randomly selected fields at $400 \times$ on a fluorescent microscope (Optiphoto II, Nikon, Tokyo, Japan). Digital images were taken using a SPOT ver.4.0.3 system (Spectracore Inc., NY, USA).

\subsection{Chemotactic effect of supernatants from PCV2- and mock-inoculated MDM on blood Mo}

To determine whether there was any soluble factor released in the culture supernatants of PCV2- and mock-inoculated MDM capable of inducing directional migration of blood Mo, a Transwell ${ }^{\circledR}$ migration assay modified from that reported by Wilflingseder et al. [43] was performed. The culture supernatants were collected from PCV2- and mock-inoculated MDM at $24 \mathrm{HPI}$. A polycarbonate membrane filter of a pore size of $5 \mu \mathrm{m}$ (Corning-Costar) was selected and immersed in RPMI-C at $37^{\circ} \mathrm{C}$ for $2 \mathrm{~h}$. The lower chamber was filled with $600 \mu \mathrm{L}$ of either culture medium or culture supernatant of PCV2- or mock-inoculated MDM collected at 24 HPI. Freshly isolated blood Mo, obtained from each corresponding pig, at $5 \times 10^{5} /$ chamber were then placed in the upper chamber. The cells in the lower chamber were harvested and counted following $4 \mathrm{~h}$ of incubation at $37{ }^{\circ} \mathrm{C}$ in $5 \% \mathrm{CO}_{2}$.

\subsection{Expression of monocytic chemokines, MIP-1 and MCP-1, by PCV2-inoculated MDM}

The expression levels of MIP-1 and MCP-1 mRNA by PCV2-inoculated MDM were measured semiquantitatively by RT-PCR as described previously $[17,22]$. For better accuracy concern, two internal controls were included as suggested by Huggett et al. [18]. The total RNA of MDM after 0 and $24 \mathrm{~h}$ of mock- or PCV2-inoculation was extracted using TRIzol reagent (Life Technologies, Paisley, Scotland, UK), reverse-transcribed to cDNA, and amplified by PCR. The primer sets used included porcine MIP-1 (sense: 5'-GCT CAG TTC AGT TCC AAG TC-3', antisense: 5'-ACC ATG AAG CTC TGC GTG AC-3') [22], porcine MCP-1 (sense: 5'-TCA CCA GCA GCA AGT GTC-3', antisense: 5'-CTG AGA TTC ACA GAG GA-3') [22], and internal controls including porcine G3PDH (sense:
5'-ACC TCC ACT ACA TGG TCT ACA TGT TC-3', antisense: 5'-CAT TGA TGA CAA GCT TCC CAT TC- $3^{\prime}$ ), and $\beta$-actin (sense: $5^{\prime}$-CAT CAC CAT CGG CAA CGA-3', antisense: $5^{\prime}$-GCG TAG AGG TCC TTC CTG ATG T-3') [3]. Amplifications were performed with a thermocycler (MJ Research, Watertown, MA, USA) using 40 cycles $\left(95^{\circ} \mathrm{C}\right.$ for $45 \mathrm{~s}, 62{ }^{\circ} \mathrm{C}$ for $45 \mathrm{~s}$, and $72{ }^{\circ} \mathrm{C}$ for $\left.1 \mathrm{~min}\right)$ for MIP-1 and MCP-1, and 40 cycles $\left(95^{\circ} \mathrm{C}\right.$ for $15 \mathrm{~s}, 60{ }^{\circ} \mathrm{C}$ for $1 \mathrm{~min}$, and $72{ }^{\circ} \mathrm{C}$ for $1 \mathrm{~min}$ ) for G3PDH and $\beta$-actin, followed by additional extension at $72{ }^{\circ} \mathrm{C}$ for $5 \mathrm{~min}$ at the end of amplification for each preparation. The PCR products were separated on a $2 \%$ agarose gel in TBE (Genmedika Biotechnology Corp., Taipei, Taiwan) by electrophoresis and stained with ethidium bromide (Sigma). The sizes of MIP-1, MCP-1, G3PDH, and $\beta$-actin are $283,375,96$, and $144 \mathrm{bp}$, respectively. The gels were photographed and analyzed using the Chemi-

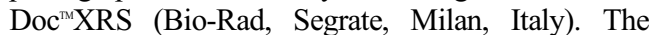
intensity of each band was normalized to the geometric mean of its corresponding G3PDH and $\beta$-actin genes and designated as the relative intensity of mRNA expression of MIP-1 or MCP-1 to compare values semi-quantitatively between the groups [18].

\subsection{Statistical analysis}

The means presented in the figures and used in the statistical analyses represent three independent experiments with 3 or 6 pigs each in triplicate per main experimental run concurrently. The data were analysed by analysis of variance (ANOVA) followed by Duncan's multiple-range test. Statistical analyses were carried out by Statistical Analysis System procedures (Statistical Analysis System; SAS for windows 6.12; SAS Institute Inc., Cary, NC, USA). A $p$ value of less than 0.05 was considered significant.

\section{RESULTS}

\subsection{Surface antigen expression}

To characterise the cell types used in the present study, the Mo and MDM were subjected to the analysis of surface antigen expression. For the freshly isolated blood Mo, there were about $74.5 \pm 15.9 \%$ of the cells expressing high levels of SWC1 (Figs. 1A and 1C) but only less than $5 \%$ of the cells were weakly positive for SWC9 (Figs. 1B and 1C). During the three days of further cultivation to allow 

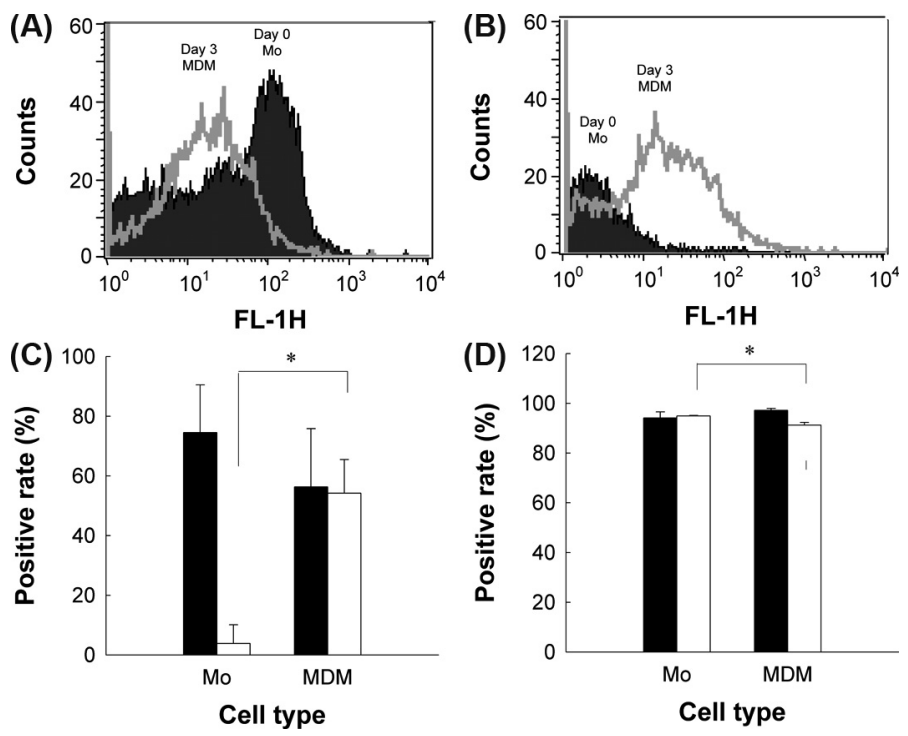

Figure 1. Characterisation of porcine blood monocytes (Mo) and monocyte-derived macrophages (MDM) by flow cytometry following staining with anti-SWC1 (A) and anti-SWC9 (B) mAb. In (A) and (B), the filled-histograms represent the Mo collected on day 0 , and the opened histograms represent the MDM after 3 days of incubation with $30 \%$ of pooled PCV1-, PCV2-, and PRRSV-free porcine plasma. The data of the positive rates of SWC1 and SWC9 (C) as well as MHC I and MHC II (D) are expressed as percentages and shown as mean \pm SD of 6 pigs from three independent experiments in triplicate. *The difference between

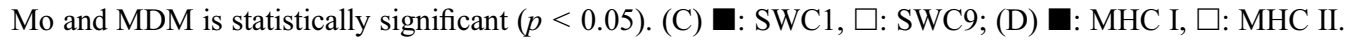

Mo transforming to MDM, the expression level of SWC1 was left-shifted in the histogram (Fig. 1A) but it was right-shifted in SWC9 (Fig. 1B). In the MDM, the SWC9 expression was rapidly and markedly up-regulated in both positivity and intensity and the positive rate reached $54.2 \pm 11.3 \%$ (Figs. $1 \mathrm{~B}$ and $1 \mathrm{C}$ ); however, the SWC1-positive rate had an approximate $20 \%$, but insignificant, down-regulation (Fig. 1C) and there was a 10-fold decrease in the SWC1 intensity (Fig. 1A). Aside from a slight but significant difference in the positive rate of MHCII between Mo (94.9 $\pm 0.2 \%)$ and MDM $(91.2 \pm 1.1 \%)$, no significant difference was seen in the positive rate of MHCI between Mo and MDM (Fig. 1D).

\subsection{Proliferation of Mo and MDM}

Microscopically, both Mo and MDM in the PCV2-inoculated group appeared to have a higher cell density than in the mock-inoculated group via the examination of the number of nuclei by Hoechst stain. To confirm the observation, Mo and MDM from both PCV2- and mockinoculated groups were collected and counted at 24-120 HPI. There was a significant reduction in the total cell count of both mock-inoculated Mo and MDM, dropping from the initial $5 \times 10^{5} /$ well and $2 \times 10^{5} /$ well to $(2.4 \pm 0.9) \times 10^{5} /$ well and $(1.2 \pm 0.2) \times 10^{5} /$ well at $24 \mathrm{HPI}$, respectively; the cell numbers then slightly reduced with time during 24-120 HPI (Figs. 2A and 2B). Conversely, although the number of PCV2-inoculated Mo dropped from $5 \times 10^{5} \%$ well to $(2.3 \pm 0.4) \times 10^{5} /$ well at $24 \mathrm{HPI}$, it increased to $(2.8 \pm 0.6) \times 10^{5} /$ well at $48 \mathrm{HPI}$ and reached the peak $(1.1 \pm 0.2) \times 10^{6} /$ well at $96 \mathrm{HPI}$ followed by a reduction to $(6.7 \pm 1.6) \times 10^{5} /$ well by 120 HPI (Fig. $\left.2 \mathrm{~A}\right)$. The PCV2-inoculated Mo had a significantly higher total cell count than that of mock-inoculated Mo at 48-120 HPI. In the PCV2-inoculated MDM, there was a slight but significant increase 

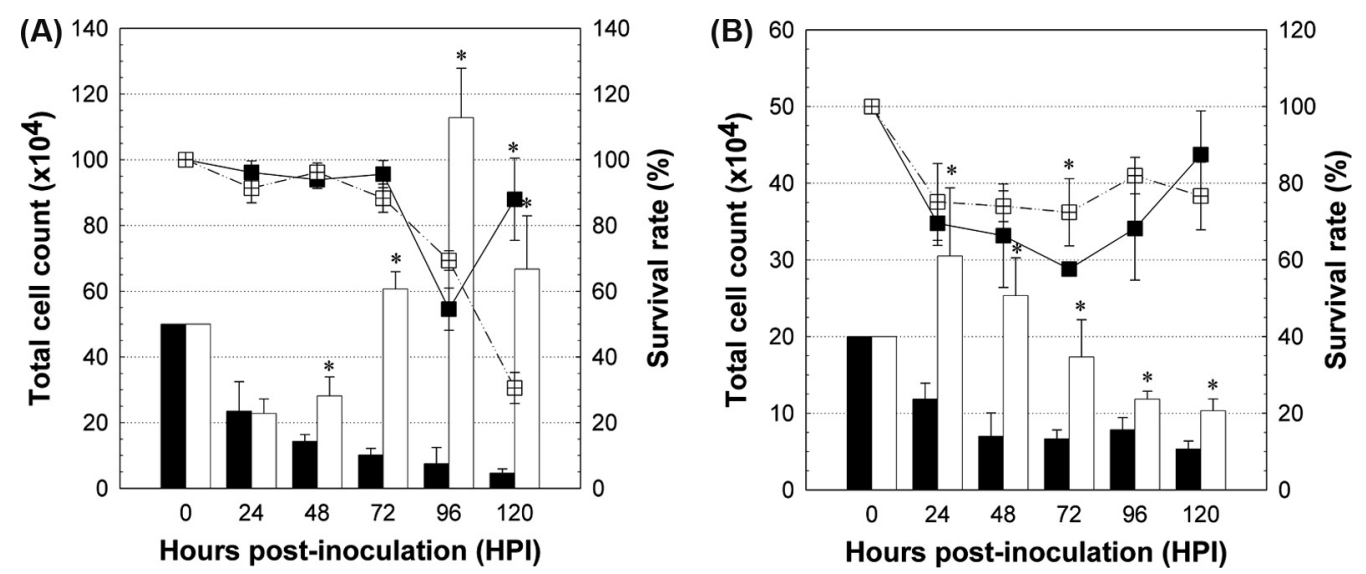

Figure 2. Changes in total cell counts and cell survival rate of mock- and porcine circovirus type 2 (PCV2)inoculated porcine monocytes (Mo) (A) and monocyte-derived macrophages (MDM) (B) over time as determined by trypan blue dye exclusion assay. Data are shown as mean \pm SD of 6 pigs from three independent experiments in triplicate. *The difference between PCV2- and mock-inoculated groups at the same hour post-inoculation (HPI) is statistically significant $(p<0.05)$. $\mathbf{\square}$ : total cell counts of mockinoculated, $\square$ : total cell counts of PCV2-inoculated, - $\square-$ : cell survival rate of mock-inoculated, $-\square-$ : cell survival rate of PCV2-inoculated.

in the cell number in the first $24 \mathrm{~h}$, reaching $(3.1 \pm 0.9) \times 10^{5} /$ well, but it then gradually reduced and dropped to $(1.0 \pm 0.2) \times 10^{5} /$ well by $120 \mathrm{HPI}$. Similar to Mo, the PCV2-inoculated MDM also showed a significantly higher total cell count than that of mock-inoculation MDM at 24-120 HPI (Fig. 2B).

\subsection{Viability}

During 24-120 HPI, the average survival rates of Mo in mock- and PCV2-inoculated groups were $54.5 \pm 6.4 \%$ to $96.2 \pm 3.5 \%$ and $30.5 \pm 4.0 \%$ to $96.2 \pm 2.8 \%$, respectively (Fig. 2A); and the average survival rates of MDM in mock- and PCV2-inoculated groups were $57.6 \pm 1.2 \%$ to $87.5 \pm 11.4 \%$ and $72.4 \pm 8.8 \%$ to $82.0 \pm 4.8 \%$, respectively (Fig. 2B). Aside from sporadic differences, no consistent findings were noted between mockand PCV2-inoculated Mo or MDM (Fig. 2).

\subsection{Formation of MGC}

To determine if PCV2 could induce the formation of MGC, the average formation rates of
MGC of both PCV2-inoculated Mo and MDM were compared with those of the corresponding mock-inoculated group at 24 HPI. In the mockinoculated MDM, spontaneous formation of MGC was observed following $24 \mathrm{~h}$ of incubation but these MGC were either bi-nucleated or tri-nucleated (Fig. 3A). On the contrary, formation of MGC with 5-8 nuclei was easily observed in the PCV2-inoculated group (Fig. 3B). Twenty-four hours after PCV2 inoculation, the MDM displayed an average fusion rate of $31.2 \pm 5.5 \%$ compared to that of $14.4 \pm 0.6 \%$ in the mock-inoculated group with a statistically significant difference. Conversely, the fusion rate was lower than $2.5 \%$ in both PCV2- and mock-inoculated Mo at $24 \mathrm{HPI}$ and no significant difference was found.

\subsection{PCV2 antigen-containing rate}

To further evaluate the role of PCV2 on the induction of MGC formation, the PCV2 antigen-containing rate was determined in Mo and MDM at $24 \mathrm{HPI}$. The positive rates in mononucleated cells and MGC were also analyzed. The Mo displayed a very low antigen-containing 

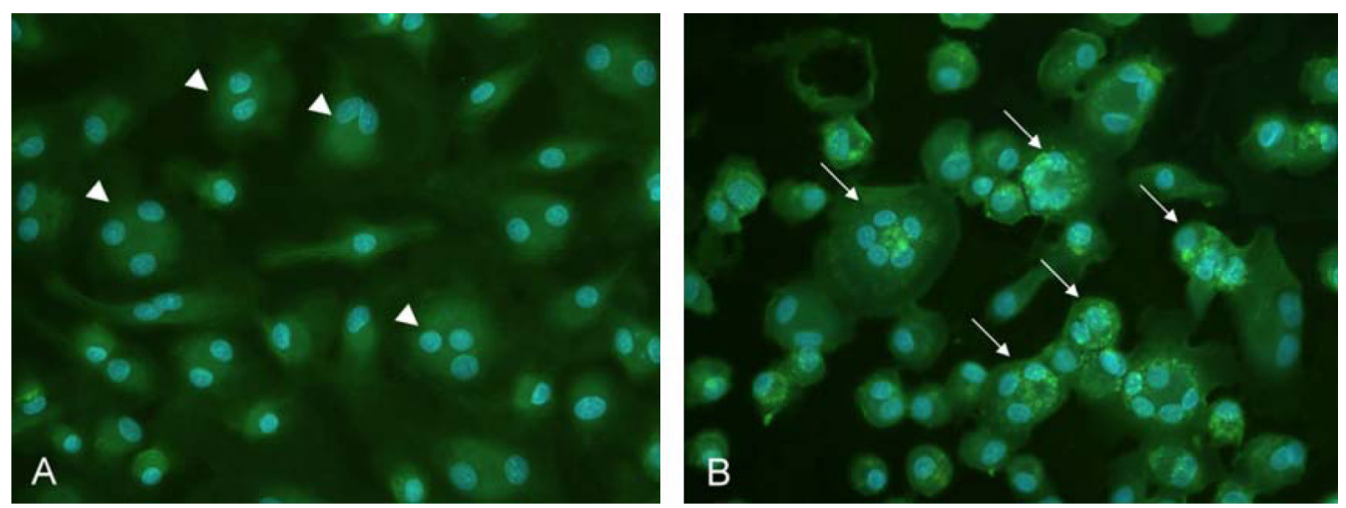

Figure 3. Porcine circovirus type 2 (PCV2)-induced cell fusion in blood monocyte-derived macrophages (MDM). Formation of multinucleated giant cells (MGC) (arrows) is compared between mock-inoculated MDM (A) and PCV2-inoculated MDM (B) as shown by co-labelling the nuclei and PCV2 antigen with Hochest stain and fluorescein isothiocyanate (FITC) by indirect immunofluorescence antibody staining, respectively. Note spontaneous cell fusion, mainly bi- and tri-nucleated, in mock-inoculated group (A, arrowheads) and significantly higher nuclear number in PCV2-inoculated group (B, arrows). (A color version of this figure is available at www.vetres.org.)

rate with an average of only $1.2 \pm 0.3 \%$, within which $82.9 \pm 11.2 \%$ and $17.1 \pm 11.2 \%$ were mono-nucleated and multinucleated Mo, respectively. On the contrary, the average antigencontaining rate of MDM reached $8.2 \pm 0.7 \%$, within which $26.6 \pm 18.5 \%$ and $73.4 \pm 18.5 \%$ were mono-nucleated and multinucleated MDM, respectively. The difference in PCV2 antigen-containing rate between MDM and Mo was statistically significant.

\subsection{Chemotactic effect of supernatants from mock- and PCV2-inoculated MDM on blood Mo}

The supernatant from the PCV2-inoculated MDM displayed a higher average chemotactic effect on the directional movement of freshly collected blood Mo after $4 \mathrm{~h}$ of incubation (Fig. 4A). When the base-line value of the control medium was subtracted, the average levels of migration activity in mock- and PCV2-inoculated groups were $107.2 \%$ and $126.8 \%$ higher than that of the control medium, respectively. However, owing to the presence of prominent individual variations in the three pigs used in the experiment (Fig. 4B), no significant difference was seen between the mock-inoculated and PCV2-inoculated groups. The supernatant of PCV2-inoculated MDM from pig 3 (female) showed evident chemotactic activity; pig 1 (male) had a slight but insignificant activity; while no such activity was seen in pig 2 (female) (Fig. 4B).

\subsection{Expression profiles of monocytic chemokines, MIP-1 and MCP-1}

The expression profiles of monocytic chemokines, MIP-1 and MCP-1, were evaluated at the level of mRNA by RT-PCR in mock- and PCV2-inoculated MDM at 0 and 24 HPI. Amplification of the template cDNA with primers specific for MIP-1 (Fig. 5A-1) and MCP-1 (Fig. 5A-2) resulted in products at sizes of $283 \mathrm{bp}$ and $375 \mathrm{bp}$, respectively. The RT-PCR products were then sequenced; comparisons with the sequences of the same MIP-1 and MCP-1 DNA segments listed in the GenBank database revealed a $99.5 \pm 0.6 \%$ and $97.3 \pm$ $2.9 \%$ of identity, respectively. Following further normalization with the corresponding house keeping genes G3PDH and $\beta$-actin, it was revealed that at 0 HPI the levels of mRNA of porcine MIP-1 and MCP-1 expressed in the mock- and PCV2-inoculated MDM were 

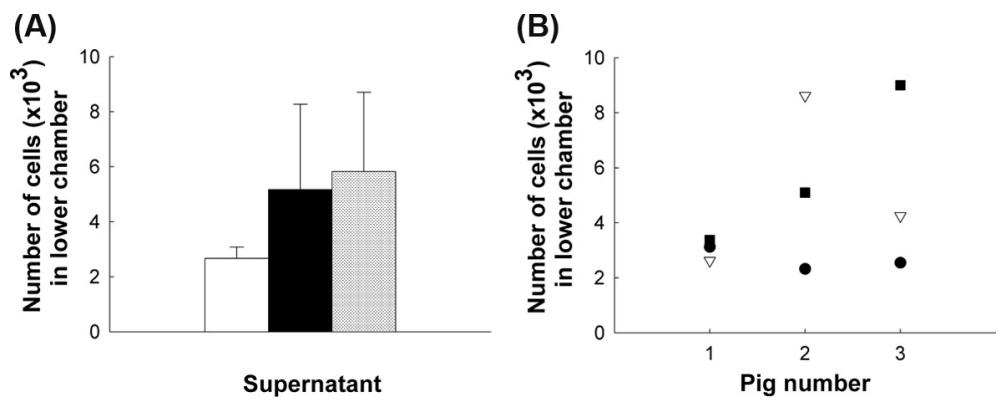

Figure 4. The chemotactic effect of the supernatants from mock- and PCV2-inoculated monocyte-derived macrophages (MDM) collected at $24 \mathrm{~h}$ post-inoculation (HPI) on blood monocytes (Mo) as determined by a migration assay using Transwell ${ }^{\circledR}$ plates. Data are expressed as (A) the total number of Mo attributed to the lower chambers shown as mean \pm SD of three pigs of the Transwell ${ }^{\circledR}$ plate and (B) dot plot of each of the three pigs used in the experiment by showing the total number of Mo attracted to the lower chamber of the Transwell ${ }^{\circledR}$ plate. (A) $\square$ : medium control, $\mathbf{\square}$ : mock-inoculated, 酯: PCV2-inoculated; (B) $\mathbf{0}$ : medium control, $\nabla$ : mock-inoculated, $\mathbf{\square}$ : PCV2-inoculated.

comparable and there were no significant differences (Fig. 5B). At 24 HPI, the levels of mRNA of porcine MIP-1 and MCP-1 expressed in PCV2-inoculated MDM were $27.8 \pm 1.5 \%$ (Fig. 5B-1) and $19.7 \pm 7.8 \%$ (Fig. 5B-2) higher than those in mock-inoculated MDM with the differences being statistically significant.

\section{DISCUSSION}

The present study demonstrates that PCV2 alone can induce proliferation, formation of MGC, and chemokine expression in the relatively more mature porcine monocytic cells, MDM, in vitro. The findings support that PCV2 alone may have a direct effect on the induction of granulomatous inflammation in vivo. It is speculated that once those PMWS-susceptible pigs are infected by PCV2, PCV2 infection-associated monocytic recruitment, proliferation, and fusion may take place in the affected organs with the subsequent development of granulomatous inflammation.

As in other animal species, porcine monocytic cells are heterogeneous and consist of subpopulations with different phenotypes and functions [2, 5, 29]. Variations of these cells in the susceptibility to different viruses or in the capacity of viral replication have been noted and the differences may be related to their phenotypes [2]. During monocyte-macrophage dif- ferentiation, the porcine Mo gradually lose their surface CD molecule, SWC1, but the amount of SWC9 is up-regulated in addition to changes in cell size and granularity [2]. When monocytic cells turn into the relatively more differentiated $\mathrm{SWC1}^{+} \mathrm{SWC9}^{+}$cells, they become susceptible to the infection of African swine fever virus [2]. Although the major role of the monocyte/ macrophage lineage cells in PCV2 infection remains controversial, these cells are generally considered as one of the major target cells for PCV2 infection in pigs based on the significant amount of PCV2 antigens and/or nucleic acid detected [9, 12, 35]. In the present study, it was revealed that under the in vitro system the freshly collected $\mathrm{SWC} 1^{\text {high }} \mathrm{SWC} 9^{\text {dim }}$ Mo had a significantly lower PCV2 antigen-containing rate than the more mature $\mathrm{SWC1}{ }^{\mathrm{dim}} \mathrm{SW}$ $\mathrm{C} 9^{\text {high }} \mathrm{MDM}$. The difference may be due to either altered cell surface receptors such as SWC9 or enhanced phagocytic ability. Whether SWC9 functions as a receptor for PCV2 attachment, facilitating PCV2 entering MDM requires further elucidation.

The results of cell proliferation and viability measurements of the present study suggest that PCV2 has a potential of enhancing proliferation and survival of Mo and MDM. This was consistent with previous finding in PCV2-inoculated alveolar macrophages (AM) [7]. It was found that PCV2 inoculation favours the survival of swine AM and PCV2-containing AM 
$(\mathrm{A}-1)$

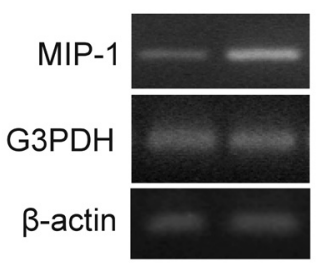

(B-1)

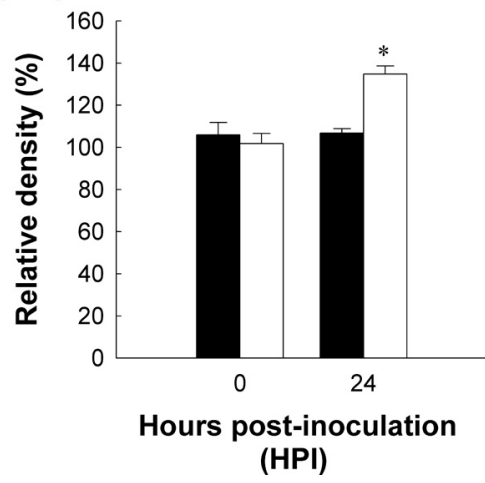

(A-2)

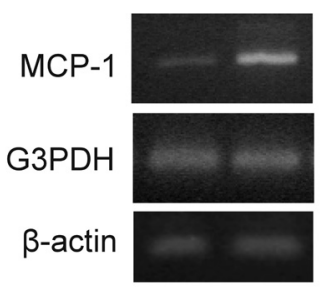

(B-2)

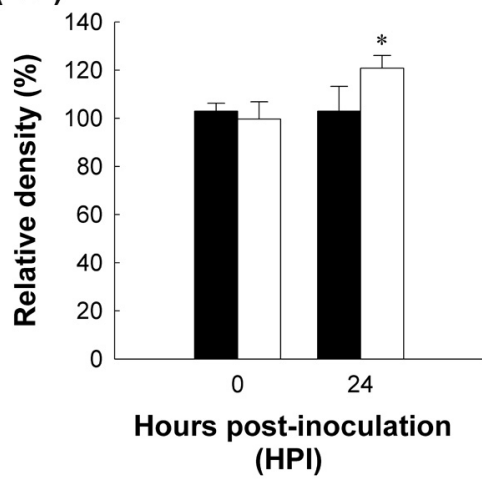

Figure 5. The mRNA expression levels of monocytic chemokines, MIP-1 and MCP-1, in mock- and PCV2-inoculated porcine monocyte-derived macrophages (MDM). The mRNA expression levels of MIP-1 and MCP-1 and the corresponding housekeeping genes G3PDH and $\beta$-actin were analyzed at 0 and $24 \mathrm{~h}$ post-inoculation (HPI) by RT-PCR and gel electrophoresis. The results of gel electrophoresis of MIP-1 (A-1) and MCP-1 (A-2) at 24 HPI and corresponding G3PDH and $\beta$-actin are displayed. The mRNA expression levels of MIP-1 (B-1) and MCP-1 (B-2) were further normalized using the geometric mean of housekeeping genes G3PDH and $\beta$-actin and the values are expressed as relative levels of mRNA expression and shown as mean $\pm \mathrm{SD}$ of three pigs from two independent experiments. *The difference between PCV2- and mock-inoculated groups is statistically significant $(p<0.05)$. Mock: MDM inoculated with an equal volume of RPMI-C; PCV2: MDM inoculated with PCV2 at an m.o.i. of 1. $\mathbf{0}$ : mockinoculated, $\square$ : PCV2-inoculated.

expressed lower apoptotic rate [7]. The present study also shows an enhanced survival tendency of Mo and MDM after PCV2 inoculation. It is speculated that PCV2 may subvert the innate immune response such as apoptosis triggered by the host against viral infection. The inhibition of apoptosis in monocytic cells by PCV2 might be essential in the persistent infection and spread of PCV2.

Lymphocyte depletion, histiocytic infiltration, and formation of MGC and cytoplasmic inclusions have been recognised as the characteristic microscopic lymphoid lesions of PMWS-affected pigs. The PMWS-affected pigs are considered to be immunosuppressed possibly due to lymphoid depletion secondary to
PCV2-induced lymphocyte apoptosis [8, 42] or decreased cell turnover rate [27]. It is known that histiocytic infiltration itself may also compromise the function of lymphoid organs [40]. However, the pathogenesis of histiocytic infiltration along with lymphoid depletion has seldom been discussed. Increases in tissue macrophages in lymphoid tissues [38, 42] and circulating blood Mo have been demonstrated in PMWSaffected pigs [33, 39]. The increased tissue macrophages, giving rise to the characteristic granulomatous nature, might attribute to either proliferation of local resident macrophages and/or infiltration of blood Mo. The present study has demonstrated a significant PCV2dependent enhancement in the proliferation of 
blood Mo and MDM. It has been reported that the number of infected tissue macrophages increased during the course of HIV-1 infection [14] and HIV-1-infected human MDM displayed enhanced macrophage-colony stimulating factor (M-CSF) production and secretion [13]. It is speculated that PCV2 infection may also create a microenvironment to promote the proliferation of monocyte/macrophage lineage cells by inducing the secretion of certain yet undetermined cytokines and/or growth factors via the activated tissue lymphocytes and/or macrophages or the PCV2-affected target cells.

Chemokines such as MIP-1 and MCP-1 have been demonstrated to co-exist with PCV2 in the same cells localised in the granulomatous lesion by in situ hybridisation; and this has been suggested to play an important role in the pathogenesis of granulomatous inflammation in PMWSaffected pigs [21, 22]. The present study has provided further evidence that PCV2 infection indeed could enhance the expression of MIP-1 and MCP-1 mRNA in MDM in vitro. In the in vitro chemotaxis assay, the supernatant from PCV2-affected MDM also showed a tendency in triggering the directional migration of blood Mo in 2 of the 3 tested pigs. The observations support that PCV2 alone could modulate the recruitment of blood Mo.

In the present study, individual variation, particularly in the chemotactic assay, was apparent. Clinically, PCV2 could be detected in pigs without clinical and/or histological evidence of PMWS as well as in PMWS-affected pigs, suggesting the presence of subclinical infections [24]. It has been suggested that whether PMWS develops in PCV2-infected pigs may be related to PCV2 capsid protein variation [26], impaired humoral immune response in which the status of the affected pigs generate insufficient neutralizing antibodies [31] or repressed cell-mediated immunity due to IL10 secretion [11]. The individual variation observed in the chemotaxis assay in the present study implies that the development of granulomatous inflammation may be dependent on the ability of PCV2-infected MDM to recruit blood Mo to the inflamed sites.

Granulomatous inflammation represents a chronic inflammatory response initiated by various infectious and non-infectious agents, but the mechanism of recruitment, activation, and maintenance of immune cells is still not clearly understood [41]. In viral infection, the formation of granulomatous lesion may be related to a stable excitement in macrophages as suggested in Ebola virus infection [37]. Activated macrophages could continue to produce cytokines, chemokines, and growth factors to exacerbate blood Mo recruitment and macrophage fusion. Macrophages are known to be important PCV2 reservoirs and this may be crucial in the pathogenesis of PCV2 infection. Formation of MGC in granulomatous lesions of PMWS-affected pigs may result from long term excitement of the PCV2-infected macrophages. Generally, the granulomatous inflammation is initiated by $\mathrm{CD}^{+} \mathrm{T}$ lymphocytes [16] and results from effector cells in response to T-cell cytokine production. The present study has demonstrated a significantly higher rate of MGC formation in PCV2-inoculated MDM in vitro without the coexistence of $\mathrm{T}$ lymphocytes. The finding further supports a direct role of PCV2 in the associated granulomatous inflammation.

M-CSF or granulocyte M-CSF (GM-CSF) could increase the formation of MGC in freshly isolated bovine Mo [44]. In addition, IL-4 [30], IL-6 [41] or interferon- $\gamma$ [4] has also been suggested to enhance the generation of MGC. Despite numerous possibilities of formation of MGC in vitro, the presence of MGC in inflammatory reactions in vivo is restricted only to relatively few viral diseases $[15,34,36]$. What direct and/or indirect role that PCV2 may play in the induction of cell fusion among MDM is an interesting and important but unanswered question.

The present study has demonstrated that PCV2 alone without the presence of other cofactors may induce proliferation of MDM and/or Mo, formation of MGC, expression of monocytic chemokines, MIP-1 and MCP-1, and directional movement of blood Mo. The results confirm that the granulomatous inflammation occurring in PMWS-affected pigs should be in close association with PCV2 infection, although individual variations may diversify the frequency and severity of this 
particular type of inflammation. Further investigation on the mechanism of formation of granulomatous inflammation in PCV2-infected pigs is currently undergoing in our laboratory.

Acknowledgements. This research was supported by Grant NSC 95-2313-B-002-062-MY3 and 98-2313B-002-025-MY3 from the National Science Council.

\section{REFERENCES}

[1] Allan G.M., Ellis J.A., Porcine circoviruses: a review, J. Vet. Diagn. Invest. (2000) 12:3-14.

[2] Basta S., Knoetig S.M., Spagnuolo-Weaver M., Allan G., McCullough K.C., Modulation of monocytic cell activity and virus susceptibility during differentiation into macrophages, J. Immunol. (1999) 162:3961-3969.

[3] Borca M.V., Gudmundsdottir I., Fernandez-Sainz I.J., Holinka L.G., Risatti G.R., Patterns of cellular gene expression in swine macrophages infected with highly virulent classical swine fever virus strain Brescia, Virus Res. (2008) 138:89-96.

[4] Byrd T.F., Multinucleated giant cell formation induced by IFN-gamma/IL-3 is associated with restriction of virulent Mycobacterium tuberculosis cell to cell invasion in human monocyte monolayers, Cell Immunol. (1998) 188:89-96.

[5] Chamorro S., Revilla C., Alvarez B., Alonso F., Ezquerra A., Dominguez J., Phenotypic and functional heterogeneity of porcine blood monocytes and its relation with maturation, Immunology (2005) 114:63-71.

[6] Chang H.W., Jeng C.R., Liu J.J., Lin T.L., Chang C.C., Chia M.Y., et al., Reduction of porcine reproductive and respiratory syndrome virus (PRRSV) infection in swine alveolar macrophages by porcine circovirus 2 (PCV2)-induced interferon-alpha, Vet. Microbiol. (2005) 108:167-177.

[7] Chang H.W., Jeng C.R., Lin T.L., Liu J.J., Chiou M.T., Tsai Y.C., et al., Immunopathological effects of porcine circovirus type 2 (PCV2) on swine alveolar macrophages by in vitro inoculation, Vet. Immunol. Immunopathol. (2006) 110:207-219.

[8] Chang H.W., Jeng C.R., Lin C.M., Liu J.J., Chang C.C., Tsai Y.C., et al., The involvement of Fas/FasL interaction in porcine circovirus type 2 and porcine reproductive and respiratory syndrome virus co-inoculation-associated lymphocyte apoptosis in vitro, Vet. Microbiol. (2007) 122:72-82.

[9] Chianini F., Majo N., Segales J., Dominguez J., Domingo M., Immunohistochemical characterisation of PCV2 associate lesions in lymphoid and non-lymphoid tissues of pigs with natural postweaning multisystemic wasting syndrome (PMWS), Vet. Immunol. Immunopathol. (2003) $94: 63-75$.
[10] D'Allaire S., Moore C., Cote G., A survey on finishing pig mortality associated with porcine circovirus diseases in Quebec, Can. Vet. J. (2007) 48:145-146.

[11] Fort M., Fernandes L.T., Nofrarias M., Diaz I., Sibila M., Pujols J., et al., Development of cell-mediated immunity to porcine circovirus type 2 (PCV2) in caesarean-derived, colostrum-deprived piglets, Vet. Immunol. Immunopathol. (2009) 129:101-107.

[12] Gilpin D.F., McCullough K., Meehan B.M., McNeilly F., McNair I., Stevenson L.S., et al., In vitro studies on the infection and replication of porcine circovirus type 2 in cells of the porcine immune system, Vet. Immunol. Immunopathol. (2003) 94:149-161.

[13] Gruber M.F., Weih K.A., Boone E.J., Smith P.D., Clouse K.A., Endogenous macrophage CSF production is associated with viral replication in HIV-1-infected human monocyte-derived macrophages, J. Immunol. (1995) 154:5528-5535.

[14] Haine V., Fischer-Smith T., Rappaport J., Macrophage colony-stimulating factor in the pathogenesis of HIV infection: potential target for therapeutic intervention, J. Neuroimmune Pharmacol. (2006) 1:32-40.

[15] Herschke F., Plumet S., Duhen T., Azocar O., Druelle J., Laine D., et al., Cell-cell fusion induced by measles virus amplifies the type I interferon response, J. Virol. (2007) 81:12859-12871.

[16] Hogan L.H., Weinstock J.V., Sandor M., TCR specificity in infection induced granulomas, Immunol. Lett. (1999) 68:115-120.

[17] Hosang K., Knoke I., Klaudiny J., Wempe F., Wuttke W., Scheit K.H., Porcine luteal cells express monocyte chemoattractant protein-1 (MCP-1): analysis by polymerase chain reaction and cDNA cloning, Biochem. Biophys. Res. Commun. (1994) 199:962-968.

[18] Huggett J., Dheda K., Bustin S., Zumla A., Realtime RT-PCR normalisation; strategies and considerations, Genes Immun. (2005) 6:279-284.

[19] James D.G., A clinicopathological classification of granulomatous disorders, Postgrad. Med. J. (2000) 76: $457-465$.

[20] Kim J., Chung H.K., Jung T., Cho W.S., Choi C., Chae C., Postweaning multisystemic wasting syndrome of pigs in Korea: prevalence, microscopic lesions and coexisting microorganisms, J. Vet. Med. Sci. (2002) 64:57-62.

[21] Kim J., Chae C., Expression of monocyte chemoattractant protein-1 but not interleukin-8 in granulomatous lesions in lymph nodes from pigs with naturally occurring postweaning multisystemic wasting syndrome, Vet. Pathol. (2003) 40:181-186.

[22] Kim J., Chae C., Expression of monocyte chemoattractant protein-1 and macrophage inflammatory protein-1 in porcine circovirus 2-induced granulomatous inflammation, J. Comp. Pathol. (2004) 131:121-126. 
[23] Krakowka S., Ellis J.A., McNeilly F., Gilpin D., Meehan B., McCullough K., Allan G., Immunologic features of porcine circovirus type 2 infection, Viral Immunol. (2002) 15:567-582.

[24] Larochelle R., Antaya M., Morin M., Magar R., Typing of porcine circovirus in clinical specimens by multiplex PCR, J. Virol. Methods (1999) 80:69-75.

[25] Larochelle R., Bielanski A., Muller P., Magar R., PCR detection and evidence of shedding of porcine circovirus type 2 in boar semen, J. Clin. Microbiol. (2000) 38:4629-4632.

[26] Larochelle R., Magar R., D’Allaire S., Genetic characterization and phylogenetic analysis of porcine circovirus type 2 (PCV2) strains from cases presenting various clinical conditions, Virus Res. (2002) 90:101-112.

[27] Mandrioli L., Sarli G., Panarese S., Baldoni S., Marcato P.S., Apoptosis and proliferative activity in lymph node reaction in postweaning multisystemic wasting syndrome (PMWS), Vet. Immunol. Immunopathol. (2004) 97:25-37.

[28] Mantovani A., Bonecchi R., Locati M., Tuning inflammation and immunity by chemokine sequestration: decoys and more, Nat. Rev. Immunol. (2006) 6:907-918.

[29] McCullough K.C., Schaffner R., Natale V., Kim Y.B., Summerfield A., Phenotype of porcine monocytic cells: modulation of surface molecule expression upon monocyte differentiation into macrophages, Vet. Immunol. Immunopathol. (1997) 58:265-275.

[30] McNally A.K., Anderson J.M., Interleukin-4 induces foreign body giant cells from human monocytes/macrophages, Differential lymphokine regulation of macrophage fusion leads to morphological variants of multinucleated giant cells, Am. J. Pathol. (1995) 147:1487-1499.

[31] Meerts P., Misinzo G., Nauwynck H.J., Enhancement of porcine circovirus 2 replication in porcine cell lines by IFN-gamma before and after treatment and by IFN-alpha after treatment, J. Interferon Cytokine Res. (2005) 25:684-693.

[32] Most J., Spotl L., Mayr G., Gasser A., Sarti A., Dierich M.P., Formation of multinucleated giant cells in vitro is dependent on the stage of monocyte to macrophage maturation, Blood (1997) 89:662-671.

[33] Nielsen J., Vincent I.E., Botner A., LadekaerMikkelsen A.S., Allan G., Summerfield A., McCullough K.C., Association of lymphopenia with porcine circovirus type 2 induced postweaning multisystemic wasting syndrome (PMWS), Vet. Immunol. Immunopathol. (2003) 92:97-111.
[34] Orenstein J.M., Wahl S.M., The macrophage origin of the HIV-expressing multinucleated giant cells in hyperplastic tonsils and adenoids, Ultrastruct. Pathol. (1999) 23:79-91.

[35] Rosell C., Segales J., Plana-Duran J., Balasch M., Rodriguez-Arrioja G.M., Kennedy S., et al., Pathological, immunohistochemical, and in-situ hybridization studies of natural cases of postweaning multisystemic wasting syndrome (PMWS) in pigs, J. Comp. Pathol. (1999) 120:59-78.

[36] Ruibal-Ares B., Riera N.E., de Bracco M.M., Macrophages, multinucleated giant cells, and apoptosis in HIV+ patients and normal blood donors, Clin. Immunol. Immunopathol. (1997) 82:102-116.

[37] Ryabchikova E., Kolesnikova L., Smolina M., Tkachev V., Pereboeva L., Baranova S., et al., Ebola virus infection in guinea pigs: presumable role of granulomatous inflammation in pathogenesis, Arch. Virol. (1996) 141:909-921.

[38] Sarli G., Mandrioli L., Laurenti M., Sidoli L., Cerati C., Rolla G., Marcato P.S., Immunohistochemical characterisation of the lymph node reaction in pig post-weaning multisystemic wasting syndrome (PMWS), Vet. Immunol. Immunopathol. (2001) 83:53-67.

[39] Segales J., Alonso F., Rosell C., Pastor J., Chianini F., Campos E., et al., Changes in peripheral blood leukocyte populations in pigs with natural postweaning multisystemic wasting syndrome (PMWS), Vet. Immunol. Immunopathol. (2001) 81:37-44.

[40] Seger R.A., Modern management of chronic granulomatous disease, Br. J. Haematol. (2008) 140:255-266.

[41] Seitzer U., Haas H., Gerdes J., A human in vitro granuloma model for the investigation of multinucleated giant cell and granuloma formation, Histol. Histopathol. (2001) 16:645-653.

[42] Shibahara T., Sato K., Ishikawa Y., Kadota K., Porcine circovirus induces B lymphocyte depletion in pigs with wasting disease syndrome, J. Vet. Med. Sci. (2000) 62:1125-1131.

[43] Wilflingseder D., Mullauer B., Schramek H., Banki Z., Pruenster M., Dierich M.P., Stoiber H., HIV-1-induced migration of monocyte-derived dendritic cells is associated with differential activation of MAPK pathways, J. Immunol. (2004) 173:7497-7505.

[44] Yoshihara K., Nagata R., Muneta Y., Inumaru S., Yokomizo Y., Mori Y., Generation of multinucleated giant cells in vitro from bovine monocytes and macrophages, J. Vet. Med. Sci. (2004) 66:1065-1069. 